\title{
OPEN A cationic surfactant-decorated liquid crystal-based sensor for sensitive detection of quinoline yellow
}

\begin{abstract}
Fatemeh S. Mohseni-Shahri ${ }^{1 \bowtie}$, Farid Moeinpour $^{1}$ \& Asma Verdian ${ }^{2}$
Quinoline yellow (OY) is one of the popular synthetic food colorants and in food industry greatly used. Developing accurate and simple QY detection procedures is of major considerable importance in ensuring food safety. Hence, it is important to detect this food colorant effectively to reduce risk. Herein, an innovative liquid crystal (LC)-based sensor was designed for the label-free and ultrasensitive detecting of the QY by means of a cationic surfactant-decorated LC interface. The nematic liquid crystal in touch with CTAB revealed a homeotropic alignment, when OY was injected into the LC-cell, the homeotropic alignment consequently altered to a planar one by electrostatic interactions between $Q Y$ and CTAB. The designed LC-based sensor detected $Q Y$ at the too much trace level as low as $0.5 \mathrm{fM}$ with analogous selectivity. The suggested LC-based sensor is a rapid, convenient and simple procedure for label-free detection of $Q Y$ in food industrial and safety control application.
\end{abstract}

Recently, there is a great enhancement in using synthetic dyes for many purposes like food additive, in cosmetics and drugs. These dyes are used to increase color, flavor and desirability in the food industries. Due to their lower sensitivity to $\mathrm{pH}$, microbial contamination and less expense of production, they have an advantage over natural dyes ${ }^{1}$.

Quinoline yellow (QY) (Fig. 1) is a synthetic yellow color dye that extensively used to give color to many food products, drugs, soft drinks, hair products and many cosmetic products. However, studies have shown that QY may cause in children skin allergies, irritation, respiratory, hyperactivity and skin rashes. There is possibility of hypersensitivity in persons who are allergenic to aspirin ${ }^{2}$. According to the premises mentioned, the European Food Safety Authority has diminished the passable intake of QY from $10 \mathrm{mg}$ to $0.5 \mathrm{mg} \mathrm{kg}^{-1}$ of body weight ${ }^{3}$. So, it is entirely worthwhile and important to design a quick, sensitive and valid procedure for the detection of QY in food stuffs.

Several analytical methods have been developed for determining of QY, including spectrophotometric ${ }^{4,5}$, spectrofluorometric $^{6}$, electrochemical methods ${ }^{7-9}$, high-performance liquid chromatography (HPLC) ${ }^{10,11}$ and UV-Vis detection ${ }^{12}$. However, these procedures are able to detect QY, but they have their own disadvantages. For example, HPLC is difficult, time-consuming and needed skilled operators, spectrophotometric method is not highly sensitive and affects by the interference of coexistent species. As well as hanging mercury drop electrode was used to QY determination, but the hanging mercury drop electrode is harmful to the environment.

In recent decade, liquid crystals (LCs) have attracted remarkable attention for the susceptible amplification and transduction of biomolecular phenomena into optical responses visible by the naked eye ${ }^{13}$. LC-based sensors have many significant benefits and do not need the use of labeled molecules and complex instrumentation ${ }^{14}$. LCs are interested materials with long-range directional order, optical anisotropy and fluidity. The alignment of LCs is extremely related to the alterations in the adjoining interface ${ }^{15}$. By LCs optical amplification, they can be turned into an incomparable optical probe for sensing reactions, such as peptide-lipid interactions, ligandreceptor binding and enzymatic reactions at the liquid crystal/aqueous interface. These interactions govern to a conversion from a homeotropic to planar orientation in the liquid crystal molecules. The liquid crystal molecules are orientated parallel and perpendicular to the surface in the planar and homeotropic alignments, respectively ${ }^{16}$. Previous researches have manifested that the respond of liquid crystal substances to exterior stimulation could be modulated by functionalizing them with surfactants, because self-assembling of the cationic surfactant at

${ }^{1}$ Department of Chemistry, Bandar Abbas Branch, Islamic Azad University, Bandar Abbas, Iran. ${ }^{2}$ Department of Food Safety and Quality Control, Research Institute of Food Science and Technology (RIFST), Mashhad, Iran. ${ }^{\bowtie}$ email: fmohsenishahri@gmail.com 


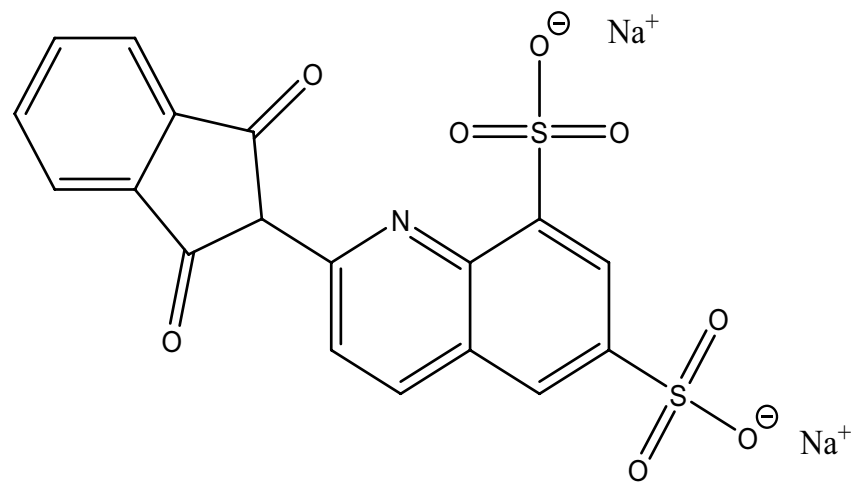

Figure 1. Chemical structure of quinoline yellow (QY).

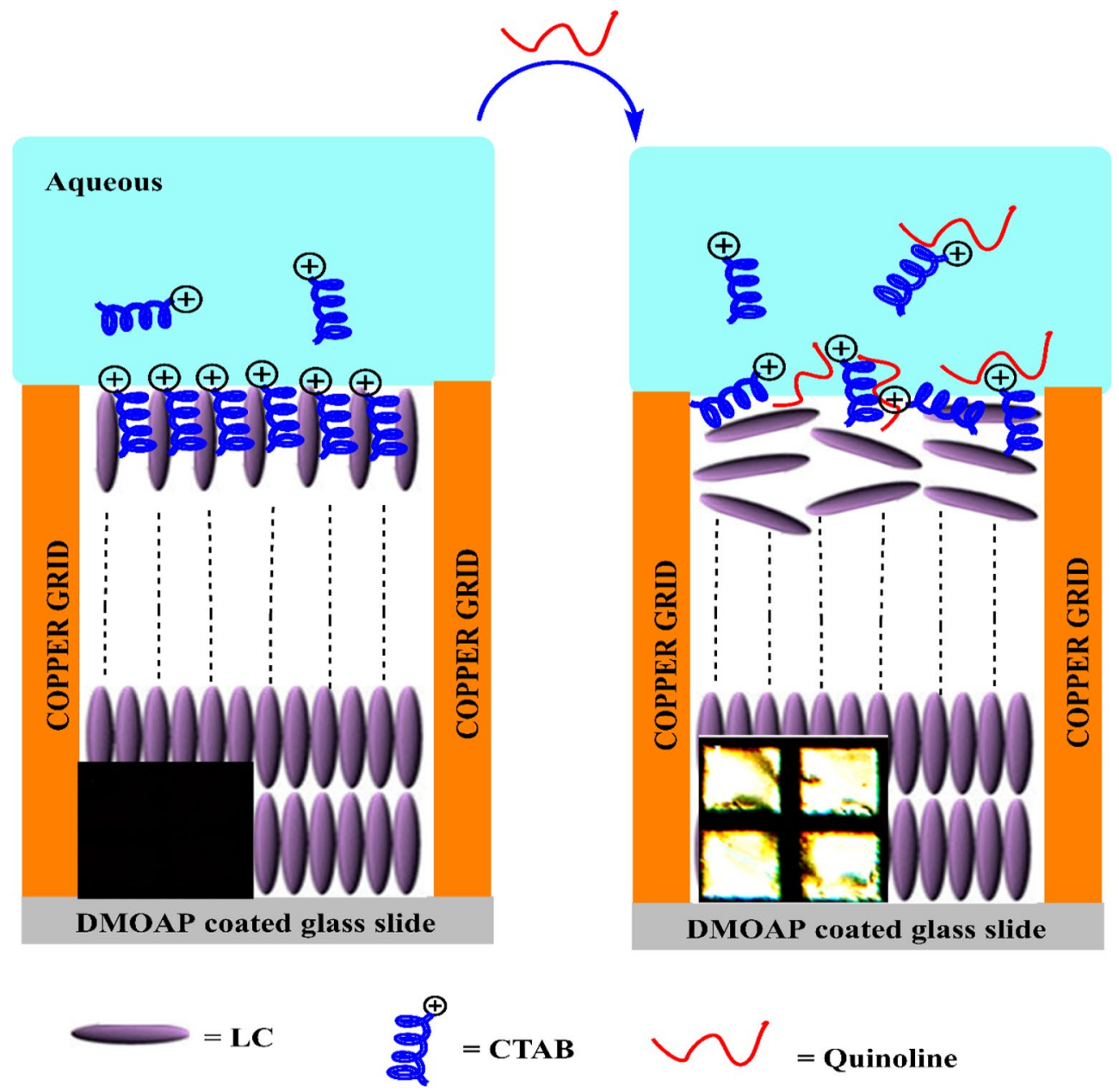

Figure 2. Schematic illustration of proposed plan for LC sensor for detection of QY based on self-assembly of $\mathrm{CTAB}$ at the interface.

the liquid crystal-aqueous interface causes homeotropic anchoring (through sidelong hydrophobic interaction between hydrocarbon chains of cationic surfactant and LC) ${ }^{17}$.

In the present research, 5CB (4-cyano-4'-pentylbiphenyl, a thermotropic nematic liquid crystal)-filled TEM copper grids were used by a cationic surfactant coverage, cetyltrimethylammonium bromide (CTAB) at the liquid crystal/aqueous interface. We expected that aqueous solution of QY would interact with CTAB, would disarrange the original orientation of liquid crystal and cause a QY detection through the homeotropic to planar orientational alert of the $5 \mathrm{CB}$ (Fig. 2). To examine this idea, we first studied interaction of CTAB and QY by 


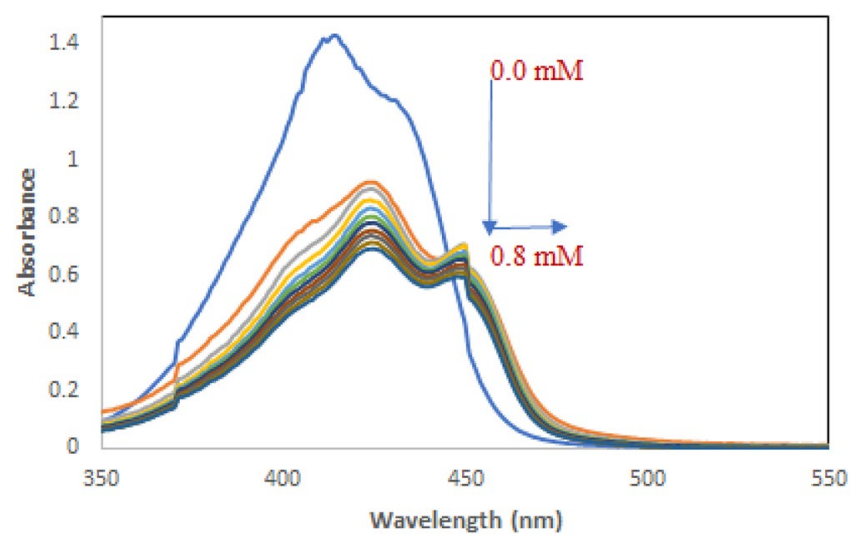

Figure 3. Ultraviolet-visible absorption spectra of QY in the presence of various concentrations of CTAB. $[\mathrm{QY}]=5.0 \mu \mathrm{M},[\mathrm{CTAB}]=4.0 \mathrm{mM}, \mathrm{T}=298 \mathrm{~K}$.

using absorption spectroscopy. Then we analyzed the limit of detection (LOD) and selectivity of this platform for QY detecting, based on the alteration in the optical pictures of liquid crystal.

\section{Materials and methods}

Materials. The standard glass microscope slides were bought from Fisher Scientific. Copper grids ( 150 mesh, $20 \mu \mathrm{m}$ in thickness and $165 \mu \mathrm{m}$ pitch), Nematic LC 4'-Pentyl-4-biphenylcarbonitrile (5CB) 98\% were purchased from Sigma-Aldrich. Cetyltrimethylammonium bromide (CTAB) were obtained from Merck. Hydrogen peroxide $30 \%$ and Sulfuric acid $99.9 \%$ purchased from Sigma-Aldrich. Quinoline yellow was obtained from Dynemic Company (India). Deionized water has been used in the preparation of all aqueous solutions.

UV-Vis spectroscopy. The UV-Vis spectra were registered at ambient temperature on a SPEKOL 1500 UV-Vis spectrophotometer supplied with $1 \mathrm{~cm}$ quartz cells. The slit width was adjusted to $5 \mathrm{~nm}$ and the wavelength range was $200-500 \mathrm{~nm}$. QY was dissolved in the DI water and diluted to $5.0 \mu \mathrm{M}$. In measuring of each data point, $50 \mu \mathrm{l}$ of the CTAB solution $(4.0 \mathrm{mM}$ ) was added to $2 \mathrm{ml}$ of the QY solution and then UV absorbance spectra were measured.

Preparation of DMOAP-coated glass substrates. The glass slides were cleansed in piranha solution $\left(30 \% \mathrm{H}_{2} \mathrm{O}_{2}\right.$ and $\left.70 \% \mathrm{H}_{2} \mathrm{SO}_{4}\right)$ at $80{ }^{\circ} \mathrm{C}$ for $2 \mathrm{~h}$ and then the slides were washed thoroughly with HPLC grade water and ethanol, several times and dried under a flow of nitrogen and drying in thermostat oven at $110^{\circ} \mathrm{C}$ for $3 \mathrm{~h}$. The cleansed glass slides were then submersed in an aqueous solution comprising $0.35 \%(\mathrm{v} / \mathrm{v})$ dimethyloctadecyl[3(trimethoxysilyl) propyl] ammonium chloride (DMOAP) for $30 \mathrm{~min}$ and washed several times with HPLC grade water and ethanol. At the end, the DMOAP-covered glass was dried again under teeny stream of nitrogen and was retained in an oven at $110^{\circ} \mathrm{C}$ for $1 \mathrm{~h}$.

Fabrication of LC-based sensor system. For the preparation of LC-sensor cell, a copper grid was put on a part of DMOAP-covered glass $(5 \mathrm{~mm} \times 5 \mathrm{~mm})$ and loaded with $2 \mu \mathrm{L}$ of $5 \mathrm{CB}$ which doped with CTAB (isotropic state at $40{ }^{\circ} \mathrm{C}$ ). Then the excess of LCs removed with a clean capillary tube. Different concentrations of QY solutions were prepared and then, QY solutions were added into the liquid crystal-based sensor platform. The optical transmission of the 5CB was determined by a polarizing optical microscope (Motic, BA 400 B-POL, Spain) in transmission mode.

Selectivity of LC sensor for QY detection. The liquid crystal-based sensor selectivity was checked with the aid of basic red 46, basic violet 16, basic yellow 28 , navy blue, disperse yellow E-3G (dis E-3G) dyes (Concentration of each sample $=50 \mathrm{fM}$ ). Data are means $\pm S D, n=3$.

\section{Results and discussion}

Investigation on the interaction between $\mathrm{QY}$ and CTAB. To investigate whether CTAB could interact with QY or not, spectrophotometric data were employed. Figure 3 shows the UV-Vis absorption spectra of QY in the presence of various concentrations of CTAB. The absorbance band intensity of QY at $484 \mathrm{~nm}$ decreased with increasing concentration of $\mathrm{CTAB}$ and the peak position shows a moderate blue shift, suggesting the formation of QY-CTAB complex. This result guide us to deduce that CTAB could willingly interact with QY to yield related complex ${ }^{18}$.

Optimization of CTAB concentration. Previous studies have shown that to regularize the alignment of the liquid crystals at the LC-aqueous interface, CTAB could be used ${ }^{19,20}$. Adding of CTAB onto the DMOAPcoated glass imparted the homeotropic regulation of liquid crystals via the hydrophobic interactions between the 


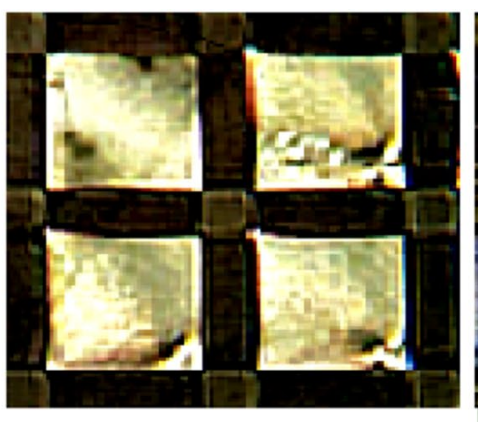

(a)

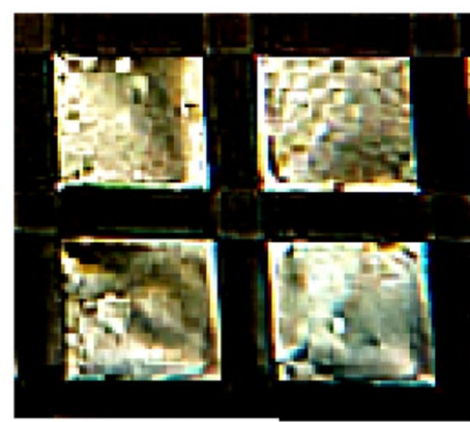

(d)

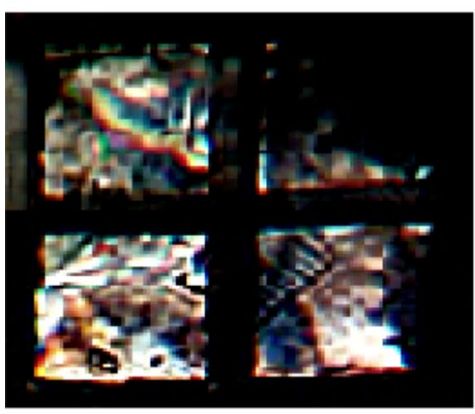

(g)

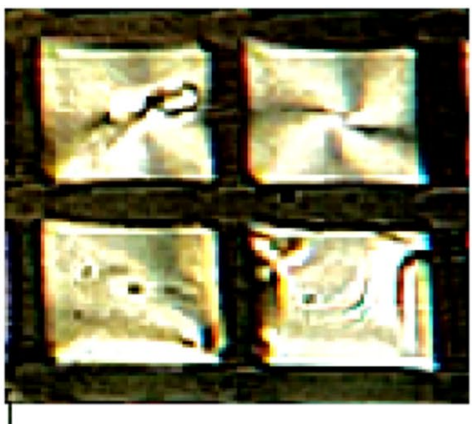

(b)

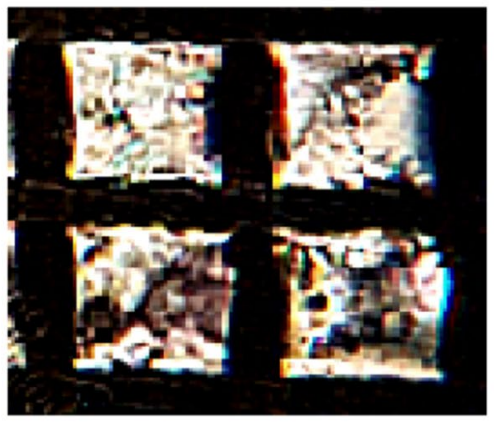

(e)

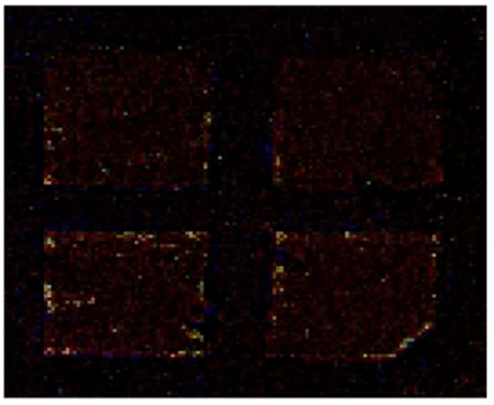

(h)

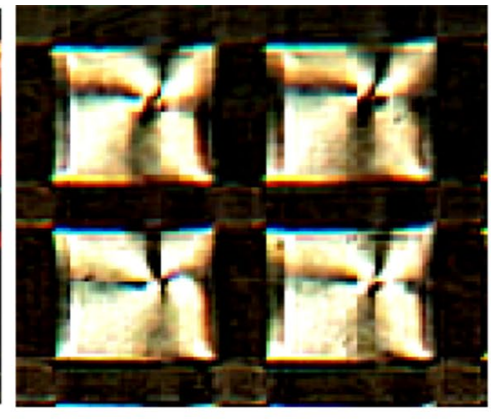

(c)

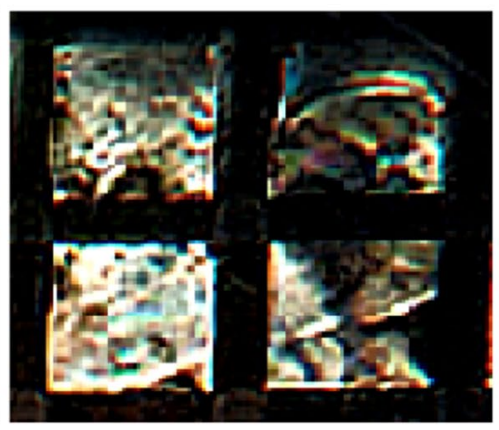

(f)

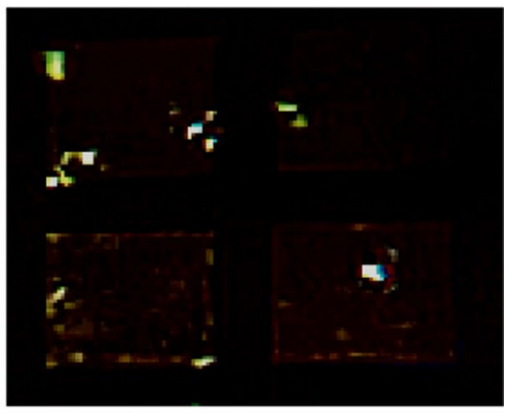

(i)

Figure 4. POM images (under crossed polarizers) of the LC cells at different concentration of $\mathrm{CTAB}=(\mathbf{a}) 0,(\mathbf{b})$ 0.5, (c) 1.0, (d) 2.0, (e) 4.0 (f) 6.0, (g) 8.0, (h) 10.0 and (i) $12.0 \mathrm{mM}$, Scale bar: $100 \mu \mathrm{m}$.

LCs and hydrocarbon chains of CTAB and causing dark optical appearances of the LCs. Hence, the minimum $\mathrm{CTAB}$ concentration adequate to operate the homeotropic anchoring of the liquid crystals should first be determined. For this reason, diverse concentrations of CTAB were injected to liquid crystal and incubated for $30 \mathrm{~min}$. Then the polarized optical pictures of liquid crystals at various concentrations of CTAB were recorded. As indicated in Fig. 4, entirely dark in appearance of liquid crystals were seen when the CTAB concentration $\geq 10 \mathrm{mM}$, corresponding to the homeotropic anchoring of the liquid crystals at the interface. Hence, a CTAB concentration of $10 \mathrm{mM}$ was considered as the optimum CTAB concentration in following experiments.

Detection of QY by using LC-based sensor. When an aqueous QY solution was inserted at the LC/ aqueous interface of liquid crystals, electrostatic interactions occurred between the anionic group of QY and cationic head groups of CTAB. The electrostatic pairing afforded the disruption of the self-assembly of CTAB at the interface and the primary homeotropic alignment of the liquid crystal molecules altered to a planar alignment. Following, we added QY solution into LC cell system to evaluate how the electrostatic interactions between CTAB and QY affect the orientations of LC and polarized optical microscope (POM) images ${ }^{15}$.

Figure 5 indicates the polarized optical microscope pictures of the LC-cell in aqueous QY solutions at various QY concentrations. The bright regions in the images become greater with the concentrations of QY increasing from 0.05 to $5 \times 10^{5} \mathrm{fM}$, confirms that the binding of QY to CTAB can change the initial homeotropic orientation of the LC-cell to a planar orientation.

To quantitatively elucidation of the developed sensor efficiency, the mean grey value parameter of the POMs was achieved by means of ImageJ software (NIH Freeware). Figure 6 demonstrates the correspondence between 


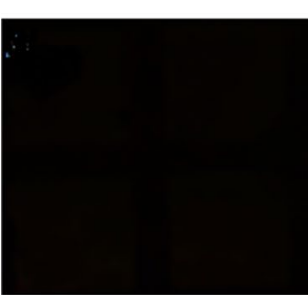

(a)

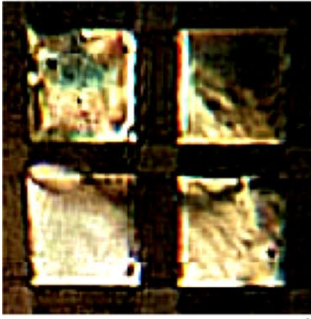

(f)

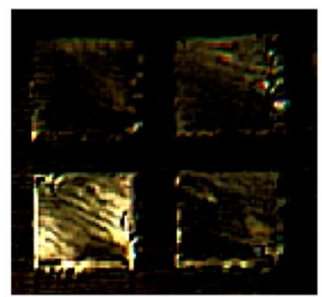

(b)

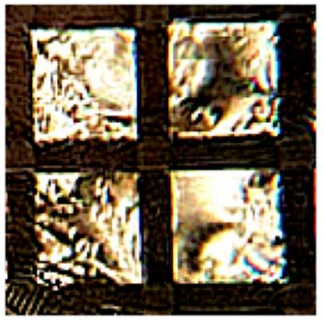

(g)

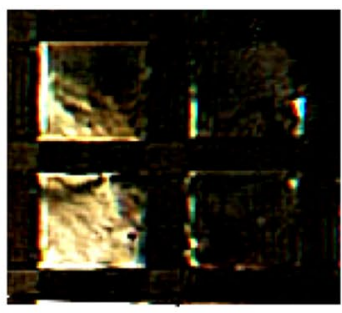

(c)

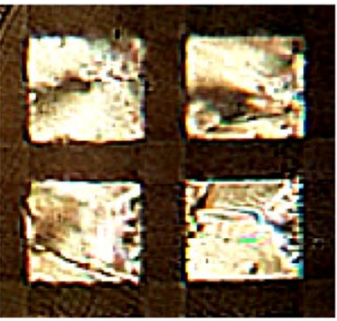

(h)

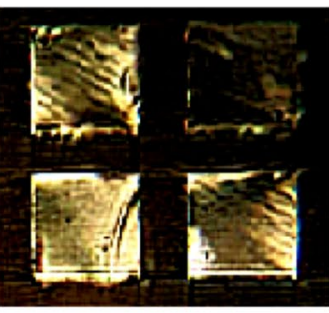

(d)

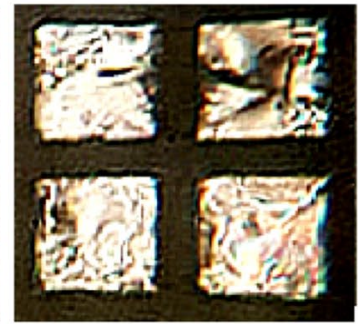

(i)

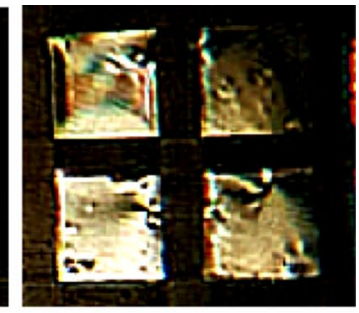

(e)

Figure 5. POM images of the CTAB-decorated aqueous/LC interfaces in contact with different concentrations of QY: (a) 0, (b) 0.05, (c) 0.5, (d) 5, (e) 50, (f) 500, (g) 5000, (h) $5 \times 10^{4}$ and (i) $5 \times 10^{5} \mathrm{fM}$, Scale bar: $100 \mu \mathrm{m}$.

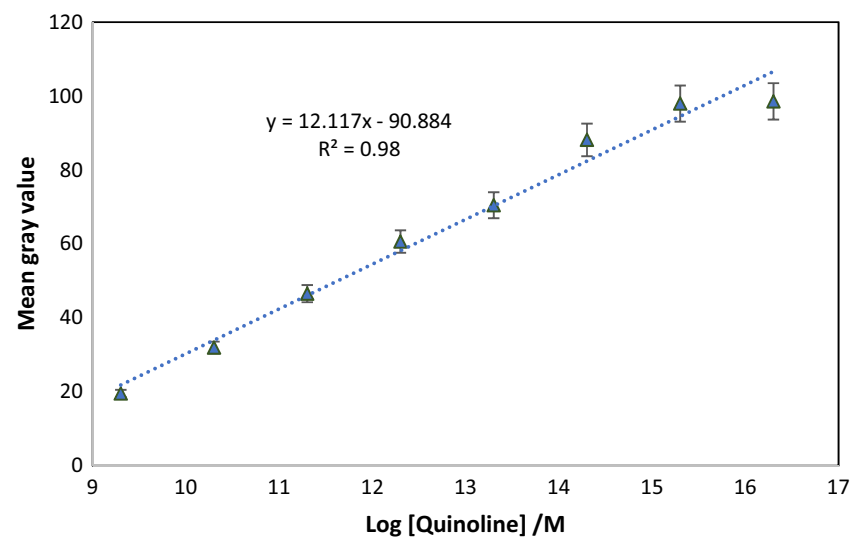

Figure 6. Graph representing mean gray value parameter as a function of QY concentration $(n=3)$.

the mean grey value and the logarithm of QY concentration. As the results show the detection limit of QY in this study is about $0.5 \mathrm{fM}$. Extraordinarily, the LC-based sensor quantitatively detected QY with a too much low detection limit.

Compared to some available sensors for the QY detection, our assessment displays relative high sensitivity with a high performance as shown in Table 1.

Selectivity of LC-based sensor for QY detection. The selectivity of the platform was assessed through basic red 46, basic violet 16, basic yellow 28, navy blue, dis E-3G dyes. The results in Fig. S1 shows that basic violet 16, basic yellow 28, navy blue and dis E-3G have no special binding to the QY sensor, therefore, the orientation of the liquid crystal molecules was conserved. Figure 7 shows the mean grey amounts of the various POMs. These outcomes confirm the selectivity of QY detection through designed liquid crystal-based sensor.

\section{Conclusion}

We reported a new and simple LC sensor based on a cationic surfactant-decorated LC interface for the detecting of QY, a hazardous food colorant. QY could disrupt the organization of the CTAB monolayer at the liquid crystal interface, therewith causing alter of the LC responses from dark-to-bright appearance. The optimum concentration for CTAB to design the sensor was $10 \mathrm{mM}$. The developed sensor has high selectivity for QY with lowest detection limit $0.5 \mathrm{fM}$, based on the alter in orientation and optical properties of the LCs for QY detection. This study introduces a simple, label-free and low-cost sensor for the detection of QY which displays promising and potential perspectives in sensing approaches. 


\begin{tabular}{|l|l|l|l|}
\hline Detection method & LOD $(\boldsymbol{\mu M})$ & Strategy & References \\
\hline Electrochemical & 1.05 & Carbon nanotube-modified electrode & 21 \\
\hline Electrochemical & 0.027 & Polyvinylpyrrolidone-modified electrode & 22 \\
\hline Electrochemical & 0.002 & Graphene oxide modified grassy carbon electrode & 23 \\
\hline Electrochemical & 0.08 & Polypyrrole/single-walled carbon nanotubes composites modified glass carbon electrode & 24 \\
\hline Electrochemical & 0.04 & Manganese dioxide functionalized graphene & 25 \\
\hline Electrochemical & 0.004 & Layer-by-layer fabricated multi-walled carbon nanotube & 26 \\
\hline Electrochemical & 0.0002 & Hanging mercury drop electrode & 27 \\
\hline Electrochemical & 40.0 & PVC-based graphite electrode & 9 \\
\hline Liquid crystal & $0.5 \times 10^{-9}$ & Homeotropic to tilted transition & This study \\
\hline
\end{tabular}

Table 1. Comparison of the reported methods for the QY detection with presented LC sensor.

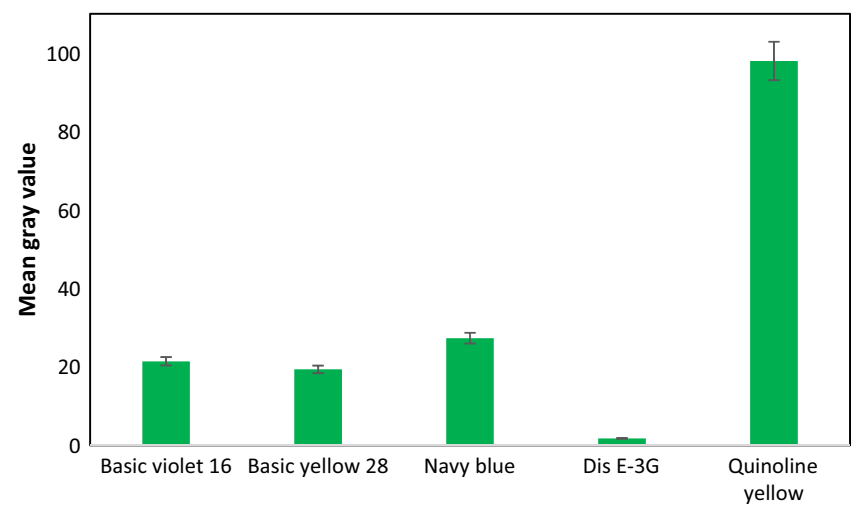

Figure 7. Selectivity of the LC-based sensor for the QY detection upon the addition of different common dyes (50 fM) under the optimal conditions.

Received: 12 August 2021; Accepted: 6 December 2021

Published online: 20 December 2021

\section{References}

1. Llamas, N. E., Garrido, M., Di Nezio, M. S. \& Band, B. S. F. Second order advantage in the determination of amaranth, sunset yellow FCF and tartrazine by UV-Vis and multivariate curve resolution-alternating least squares. Anal. Chim. Acta 655, 38-42 (2009).

2. Khan, M. S. et al. Food additive dye (quinoline yellow) promotes unfolding and aggregation of myoglobin: A spectroscopic and molecular docking analysis. Spectrochim. Acta A 214, 216-226 (2019).

3. Scientific Opinion on the re-evaluation of quinoline yellow (E 104) as a food additive 1 EFSA panel on food additives and nutrient sources added to food (ANS). EFSA J. 7, 1329 (2009)

4. Ozgur, M. U. \& Koyuncu, I. The simultaneous determination of quinolone yellow (E-104) and sunset yellow (E-110) in syrups and tablets by second derivative spectrophotometry. Turk. J. Chem. 26, 501-508 (2002).

5. Capitan-Vallvey, L. F., Valencia, M. C. \& Nicolas, E. A. Flow injection analysis with in-line solid phase extraction for the spectrophotometric determination of sulfonated and unsulfonated quinoline yellow in cologne. Fresenius J. Anal. Chem. 367, 672-676 (2000).

6. Shahabadi, N., Maghsudi, M. \& Rouhani, S. Study on the interaction of food colourant quinoline yellow with bovine serum albumin by spectroscopic techniques. Food Chem. 135, 1836-1841 (2012).

7. Zhang, S., Shi, Z. \& Wang, J. Sensitive and rapid determination of quinolone yellow in drinks using polyvinylpyrrolidone-modified electrode. Food Chem. 173, 449-453 (2015).

8. Sakac, N., Regusic, L., Sak-Bosnar, M., Jozanovic, M. \& Breslauer, N. Direct potentiometric determination of ptyalin in saliva. Int. J. Electrochem. Sci. 9, 7097-7109 (2014).

9. Rouhani, S. \& Haji-ghasemi, T. Novel PVC-based coated graphite electrode for selective determination of quinoline yellow. J. Iran. Chem. Soc. 6, 679-685 (2009).

10. Khanavi, M. et al. Development of a green chromatographic method for simultaneous determination of food colorants. Food Anal. Methods 5, 408-415 (2012).

11. Zou, T., He, P., Yasen, A. \& Li, Z. Determination of seven synthetic dyes in animal feeds and meat by high performance liquid chromatography with diode array and tandem mass detectors. Food Chem. 138, 1742-1748 (2013).

12. Oka, H. et al. Purification of quinoline yellow components using high-speed counter-current chromatography by stepwise increasing the flowrate of the mobile phase. J. Chromatogr. A 989, 249-255 (2003).

13. Park, S. J. \& Jang, C. H. Using liquid crystals to detect DNA hybridization on polymeric surfaces with continuous wavy features. Nanotechnology 21, 425502 (2010).

14. Yasuhara, K., Sasaki, Y. \& Kikuchi, J. I. Fluorescent sensor responsive to local viscosity and its application to the imaging of liquidordered domain in lipid membranes. Colloids Surf. B 67, 145-149 (2008).

15. Munir, S. \& Park, S. Y. Liquid crystal-Based DNA biosensor for myricetin detection. Sens. Actuators B Chem. 233, 559-565 (2016).

16. Khan, M. \& Park, S. Y. Liquid crystal-based proton sensitive glucose biosensor. Anal. Chem. 86, 1493-1501 (2014). 
17. Omer, M. et al. Biosensor utilizing a liquid crystal/water interface functionalized with poly (4-cyanobiphenyl-4'-oxyundecylacrylateb-((2-dimethyl amino) ethyl methacrylate)). Colloids Surf. B 121, 400-408 (2014).

18. Li, Z. et al. Identification of the binding between three fluoronucleoside analogues and fat mass and obesity-associated protein by isothermal titration calorimetry and spectroscopic techniques. J. Pharm. Biomed. Anal. 149, 290-295 (2018).

19. Verma, I., Devi, M., Sharma, D., Nandi, R. \& Pal, S. K. Liquid crystal based detection of Pb(II) ions using spinach RNA as recognition probe. Langmuir 35, 7816-7823 (2019).

20. Nguyen, D. K. \& Jang, C. H. Label-free liquid crystal-based detection of As(III) ions using ssDNA as a recognition probe. Microchem. J. 156, $10483(2020)$.

21. Zhao, J., Zhang, Y., Wu, K. B., Chen, J. W. \& Zhou, Y. K. Electrochemical sensor for hazardous food colorant quinoline yellow based on carbon nanotube modified electrode. Food Chem. 128, 569-572 (2011).

22. Zhang, S., Shi, Z. \& Wang, J. Sensitive and rapid determination of quinoline yellow in drinks using polyvinylpyrrolidone-modified electrode. Food Chem. 173, 449-453 (2015).

23. Fu, L., Zheng, Y., Wang, A., Cai, W. \& Lin, H. Sensitive determination of quinoline yellow using poly (diallyldimethylammonium chloride) functionalized reduced graphene oxide modified grassy carbon electrode. Food Chem. 181, 127-132 (2015).

24. Gao, Y., Wang, M., Yang, X., Sun, Q. \& Zhao, J. Rapid detection of quinoline yellow in soft drinks using polypyrrole/single-walled carbon nanotubes composites modified glass carbon electrode. J. Electroanal. Chem. 735, 84-89 (2014).

25. Wu, Y. et al. Construction of effective electrochemical sensor for the determination of quinoline yellow based on different morphologies of manganese dioxide functionalized graphene. J. Food Compos. Anal. 84, 103280 (2019).

26. Zheng, Y., Fu, L., Wang, A. \& Cai, W. Electrochemical detection of quinoline yellow in soft drinks based on layer-by-layer fabricated multi-walled carbon nanotube. Int. J. Electrochem. Sci. 10, 3530-3538 (2015).

27. Nevado, J. J. B., Flores, J. R., Llerena, M. J. V. \& Farinas, N. R. Rapid spectrophotometric method to resolve ternary mixtures of Tartrazine, Quinoline Yellow and Patent Blue V in commercial products. Fresenius J. Anal. Chem. 365, 383-388 (1999).

\section{Acknowledgements}

The authors are grateful for the financial support of the Islamic Azad University Bandar Abbas Branch for this study.

\section{Author contributions}

F.S.M.-S.: Investigation, Supervision and writing-original draft, F.M.: Conceptualization, Methodology, Writing-review, Drawing Figures and editing, A.V.: Conceptualization, Project administration, Writing-review and editing.

\section{Competing interests}

The authors declare no competing interests.

Additional information

Supplementary Information The online version contains supplementary material available at https://doi.org/ 10.1038/s41598-021-03788-6.

Correspondence and requests for materials should be addressed to F.S.M.-S.

Reprints and permissions information is available at www.nature.com/reprints.

Publisher's note Springer Nature remains neutral with regard to jurisdictional claims in published maps and institutional affiliations.

(c) (i) Open Access This article is licensed under a Creative Commons Attribution 4.0 International License, which permits use, sharing, adaptation, distribution and reproduction in any medium or format, as long as you give appropriate credit to the original author(s) and the source, provide a link to the Creative Commons licence, and indicate if changes were made. The images or other third party material in this article are included in the article's Creative Commons licence, unless indicated otherwise in a credit line to the material. If material is not included in the article's Creative Commons licence and your intended use is not permitted by statutory regulation or exceeds the permitted use, you will need to obtain permission directly from the copyright holder. To view a copy of this licence, visit http://creativecommons.org/licenses/by/4.0/.

(c) The Author(s) 2021 